\title{
The role of CPAP treatment in elderly patients with moderate obstructive sleep apnoea: a multicentre randomised controlled trial
}

\author{
Silvia Ponce ${ }^{1}$, Esther Pastor², Belen Orosa ${ }^{1}$, Grace Oscullo $\mathbb{1}^{3}$, Pablo Catalán ${ }^{4}$, \\ Alfonso Martinez ${ }^{5}$, Luis Hernández ${ }^{6}$, Alfonso Muriel ${ }^{7}$, Eusebi Chiner ${ }^{2}$ and \\ Miguel Ángel Martínez-García ${ }^{3}$ on behalf the Sleep Respiratory Disorders \\ Group of the Sociedad Valenciana de Neumología
}

@ERSpublications

CPAP treatment improves diurnal hypersomnia and some sleep-related symptoms and quality of life in elderly patients with moderate obstructive sleep apnoea http://bit.ly/2EvjSGn

Cite this article as: Ponce S, Pastor E, Orosa B, et al. The role of CPAP treatment in elderly patients with moderate obstructive sleep apnoea: a multicentre randomised controlled trial. Eur Respir J 2019; 54: 1900518 [https://doi.org/10.1183/13993003.00518-2019].

ABSTRACT The efficacy of continuous positive airway pressure (CPAP) treatment in elderly patients with nonsevere obstructive sleep apnoea (OSA) is controversial. The objective of this study was to assess the effect of CPAP treatment in elderly patients with moderate OSA in terms of clinical, quality-of-life and neurocognitive measures.

This was an open-label, randomised, multicentre clinical trial in 145 elderly patients ( $\geqslant 70$ years old) with confirmed moderate OSA (apnoea-hypopnoea index 15-29.9 events $\mathrm{h}^{-1}$ ) randomised to receive CPAP $(n=73)$ or no CPAP $(n=72)$ for 3 months. The primary end-point was the Epworth Sleepiness Scale (ESS) score, and the secondary end-points included quality of life (Quebec Sleep Questionnaire (QSQ) domain scores), sleep-related symptoms, presence of anxiety/depression, office-based blood pressure measurements and some neurocognitive tests. The analysis was performed according to the intention-to-treat principle.

Mean \pm SD age was $74.9 \pm 4.6$ years. The CPAP group achieved a greater improvement in the ESS score (adjusted difference of 2.6 (95\% CI 3.6-1.6) points; effect size 1) in some sleep-related symptoms and in some dimensions of the QSQ questionnaire (nocturnal symptoms: -0.7 (95\% CI $-0.3--1.0$ ) points; $\mathrm{p}<0.0001$ and emotions: $-0.4(95 \%$ CI $-0.1--0.7)$ points; $\mathrm{p}=0.023)$. However, CPAP did not demonstrate any effect on either neurocognitive tests (including anxiety and depression) or blood pressure levels. There was a positive correlation between the effect of CPAP and the improvement in ESS scores and quality of life domains.

CPAP treatment resulted in a significant improvement in diurnal hypersomnia and some sleep-related symptoms and quality of life domains in elderly patients with moderate OSA.

\footnotetext{
This article has supplementary material available from erj.ersjournals.com

This study is registered at ClinicalTrials.gov with identifier number NCT03079466. Individual participant data that underlie the results reported in this article will be shared, after de-identification, beginning 3 months and ending 24 months following article publication, for those investigators whose proposed use of the data has been approved by an independent review committee identified for this purpose and in order to achieve aims in the approved proposal. Proposals should be directed to mianmartinezgarcia@gmail.com
}

Received: March 122019 | Accepted after revision: May 172019

Copyright OERS 2019 


\section{Introduction}

Obstructive sleep apnoea (OSA) is one of the most common diseases in the general population and its prevalence is expected to rise in the future, for various reasons: the endemic nature of obesity, greater access to diagnostic techniques and increased life expectancy $[1,2]$. The prevalence of OSA in the elderly is higher than that in the middle-aged population, but little is known about the effect of OSA and continuous positive airway pressure (CPAP) on the elderly population [3, 4]. This is basically because there is a physiological increase in the number of sleep-related respiratory events with age [5]. However, the cut-off point on the apnoea-hypopnoea index (AHI) that distinguishes the physiological from the pathological in the elderly is not know, although it is likely higher than AHI $\geqslant 5$ events $\cdot h^{-1}$ that is habitually used to define OSA [5]. It is therefore difficult to establish which elderly patients will benefit from CPAP treatment, especially in nonsevere forms of OSA.

A recent clinical trial undertaken by our group demonstrated the efficacy of 3 months of CPAP in terms of both clinical findings and quality of life in patients aged $\geqslant 70$ years with severe OSA (AHI $\geqslant 30$ events.h $^{-1}$ ) [6]. Similarly, another clinical trial reported a positive effect of 12 months of CPAP treatment on hypersomnia, but not on quality of life, in patients aged $\geqslant 65$ years with symptomatic OSA (Epworth Sleepiness Scale (ESS) score $\geqslant 9$ ) [7]. In the light of these results, the main objective of the present study was to analyse the effect of CPAP in patients aged $\geqslant 70$ years referred to sleep units on suspicion of OSA in whom moderate OSA was diagnosed by respiratory polygraphy or full polysomnography (PSG).

\section{Methods}

\section{Design overview and setting}

This was an open-label, randomised, multicentre clinical trial of parallel groups with a blinded end-point design, conducted in six teaching clinical centres in Spain in elderly patients referred to sleep laboratories because of clinical suspicion of OSA and diagnosed with moderate OSA. Patients were randomly assigned to either CPAP or no therapy for 3 months.

The study was approved by the ethics committee of each participating centre. All participants provided informed signed consent. The study's quality was evaluated according to the Consolidated Standards of Reporting Trials (CONSORT) items. The trial was registered at ClinicalTrials.gov with identifier number NCT03079466.

\section{Participants}

Consecutive patients referred to the sleep laboratories due to clinical suspicion of OSA were recruited from the participating centres. Patients were initially eligible for participation in the study if they were aged $\geqslant 70$ years and had AHI 15-29.9 events $\cdot \mathrm{h}^{-1}$ [8]. Exclusion criteria included current use of CPAP treatment, central sleep apnoea (defined as at least $50 \%$ of respiratory events with a pattern of apnoea or hypopnoea without any respiratory effort), respiratory failure (defined as diurnal oxygen saturation <90\%), severe heart failure (New York Heart Association functional class III-IV), a cardiovascular event in the month prior to inclusion in the study, disabling hypersomnia (ESS score $\geqslant 18$ ) and previous severe impairment of cognitive performance that, in the opinion of the researchers, ruled out their inclusion in the study.

\section{Procedures}

Sleep study and CPAP pressure titration

Each participant was subjected to a sleep study, either full standard PSG (Alice 5; Respironics, Murrysville, PA, USA) or respiratory polygraphy (Alice PDx; Respironics) with a device previously validated against PSG. All data were interpreted manually. We followed the Spanish Society of Pneumology and Thoracic Surgery guidelines for the diagnosis and treatment of OSA (see the supplementary material for more information) [9].

\section{Initial visit}

At the initial visit after the sleep study, all the patients completed a standardised protocol that included general and anthropometric data, personal history especially related to cardiovascular, depression, anxiety

Affiliations: ${ }^{1}$ Pneumology Dept, Hospital Universitario Dr Peset, Valencia, Spain. ${ }^{2}$ Pneumology Dept, Hospital Universitario San Juan, Alicante, Spain. ${ }^{3}$ Pneumology Dept, Hospital Universitario y Politécnico La Fe, Valencia, Spain. ${ }^{4}$ Internal Medicine Dept, Hospital General de Requena, Valencia, Spain. ${ }^{5}$ Pneumology Dept, Hospital General Universitario de Castellón, Valencia, Spain. ${ }^{6}$ Pneumology Dept, Hospital General Universitario de Alicante, Valencia, Spain. ${ }^{7}$ Clinical Biostatistics Unit, Hospital Universitario Ramón y Cajal, IRYCIS, CIBERESP, Nursing Dept, Alcala University, Madrid, Spain.

Correspondence: Miguel Ángel Martínez-García, Pneumology Dept, Hospital Universitario and Politécnico La Fe, Bulevar Sur s/n, 46012 Valencia, Spain. E-mail: mianmartinezgarciadagmail.com 
or other neurocognitive diseases, current medication, and clinical history related to OSA, including chronic snoring, witnessed apnoeas, daytime hypersomnolence, nightmares, choking and nocturia (based on the patient's report that they would wake up to go to the toilet at least 2 times during the night). The validated Spanish version of the ESS was used to quantify daytime somnolence [10].

All patients completed a specific sleep-related quality of life questionnaire: the validated Spanish language version of the Quebec Sleep Questionnaire (QSQ). The QSQ includes the quantification of diurnal and nocturnal symptoms, hypersomnolence, and social interactions and emotional aspects [11, 12]. Cognitive function was assessed by an extensive neuropsychological battery administered at the same time of day in each evaluation $(09: 00 \mathrm{~h})$ by trained, blinded personnel; this covered subjective sleepiness, executive function, visual attention, speed of processing and mental flexibility (Trail Making Test (TMT) A and B) [13], working memory (digit symbol and digit span tests) [14], and symptoms of anxiety or depression (Hospital Anxiety and Depression Scale (HADS)) [15]. Finally, three office-based blood pressure measurements were taken, following international recommendations [16].

\section{Follow-up}

The follow-up period was 3 months, during which the patients (with or without CPAP) had direct contact with the research team at all times for the resolution of clinical problems or doubts related to the study. Medical appointments were scheduled for all patients at 4 and 12 weeks after randomisation to ensure the same number of medical visits in all patients (with or without CPAP). Every medical appointment involved protocol-based assessments of the following: adherence to CPAP and side-effects (for the CPAP group) and changes in treatment, and re-evaluation of the exclusion criteria. At the last medical appointment, after 12 weeks, all the patients again completed a standardised protocol that included sleep-related symptoms, neurocognitive tests, QSQ and blood pressure measurements taken at the same time as the pre-randomisation tests. Adherence to CPAP was always objectively assessed by reading the device's time counter from the start of treatment to the end of the follow-up. Patients were classified as being adequately treated with CPAP if the treatment had started and the average cumulative adherence was $\geqslant 4 \mathrm{~h} \cdot$ day $^{-1}$.

\section{Primary and secondary outcomes}

The primary outcome was the change (from the beginning of the study to the end of follow-up) in the ESS score. Secondary outcomes included changes in quality of life (QSQ domain scores), sleep-related symptoms, anxiety/depression, office-based blood pressure measurements and some neurocognitive tests.

\section{Statistical analysis}

Continuous variables are expressed as mean with standard deviation or median (interquartile range), while categorical variables are reported as absolute numbers and percentages.

The sample size was calculated on the basis of a clinically significant change in the primary end-point (ESS score) of 2 points (based on the results of McMillan et al. [7]), and assuming an $\alpha$ error of 5\%, a statistical power of $80 \%$ and $10 \%$ withdrawals during the follow-up, with a result of 71 patients needed per randomised arm. The analysis was performed according to the intention-to-treat (ITT) principle.

Patients with AHI 15-29.9 events. $\mathrm{h}^{-1}$ were eligible for randomisation by software specifically designed to allocate groups for the patients. Random allocation stratified by site was used, without any other restriction. The software only revealed the allocation group when an investigator provided the data of a fully eligible patient, which guaranteed concealment of the randomisation sequence.

The intragroup differences from the beginning to the end of the study were evaluated with the paired t-test and the intergroup comparisons were assessed by ANCOVA, with adjustment for the corresponding baseline variable of the outcome. The Chi-squared test was used to compare dichotomous variables. The effect size was estimated by dividing this difference by the standard deviation of the baseline measurement. As proposed by COHEN [17], an effect size of $0.20,0.50$ and 0.80 was considered as small, medium and large, respectively. Correlation between quantitative variables was analysed using the Pearson test. Logistic regression analysis was used to estimate the odds ratio and $95 \%$ confidence interval of having clinical symptoms (as dichotomous variables) and/or ESS score $\geqslant 10$ in the CPAP group compared with the control group without CPAP, adjusting for baseline status.

A two-sided p-value $<0.05$ was considered significant. A multiple imputation method was implemented in 10 patients with missing follow-up data in the ESS score $(n=3)$ or QSQ domain scores $(n=7)$, under the assumption that these data were missing at random. Stata version 11 (StataCorp, College Station, TX, USA) and SPSS version 21 (IBM, Armonk, NY, USA) were used for analysis. 


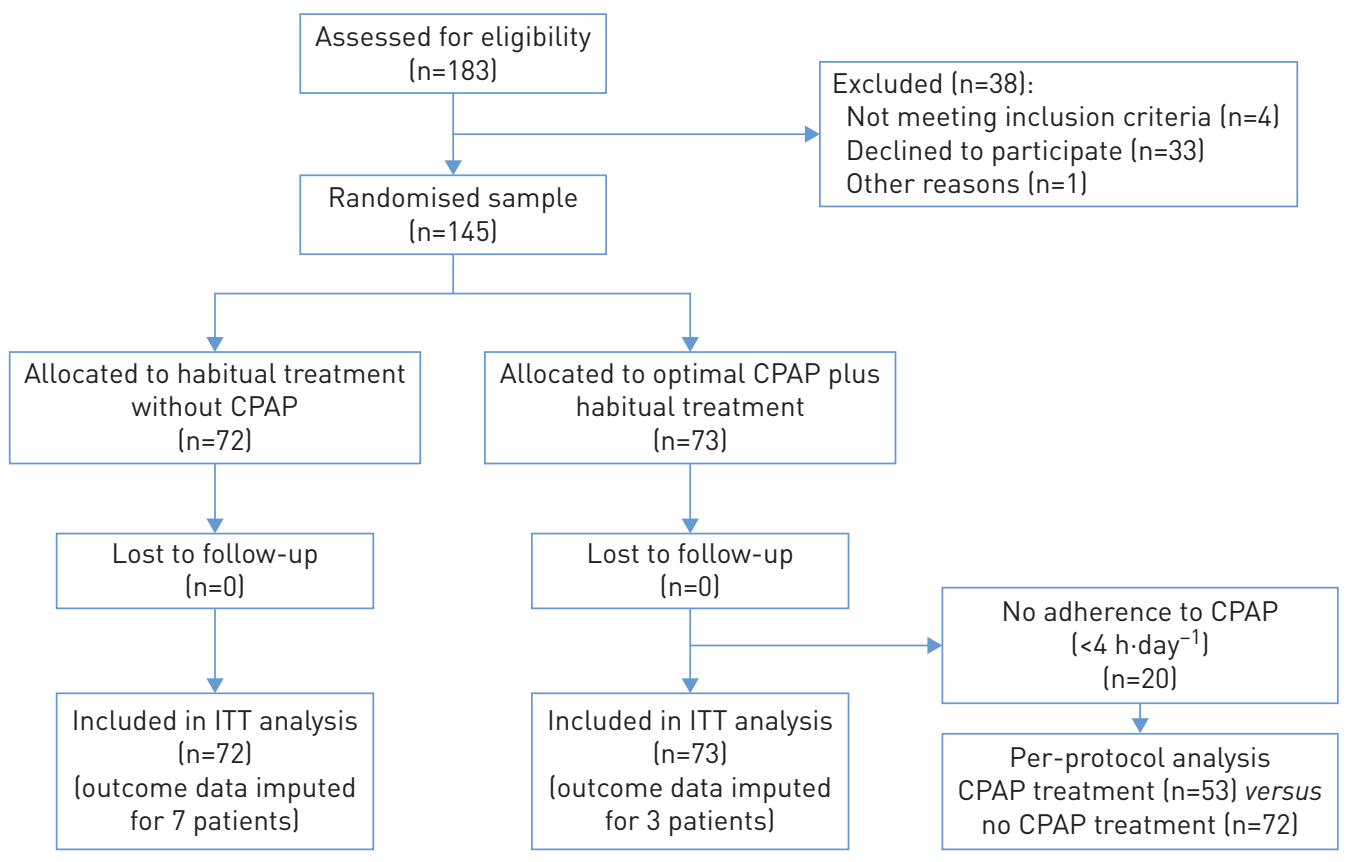

FIGURE 1 Flowchart of the study. CPAP: continuous positive airway pressure; ITT: intention-to-treat.

\section{Results}

183 patients were initially recruited between March 2017 to July 2017 and 145 were randomised: 73 to the CPAP arm and 72 to the no CPAP (control) arm (figure 1). No statistical or clinical differences were observed between the randomised groups (table 1). Mean \pm SD age was $74.9 \pm 4.6$ years; $87(60.8 \%)$ patients were male; mean \pm SD body mass index (BMI) was $30.5 \pm 5.5 \mathrm{~kg} \cdot \mathrm{m}^{-2} .37 .8 \%$ had an ESS score $\geqslant 10$.

\section{TABLE 1 Baseline characteristics of randomised patients}

\begin{tabular}{|c|c|c|c|}
\hline & All patients & CPAP treatment & No CPAP treatment \\
\hline Subjects & 145 & 73 & 72 \\
\hline Age years & $74.9 \pm 4.6$ & $74.6 \pm 4.2$ & $75.0 \pm 5.0$ \\
\hline Males & $95(65.5)$ & $50(68.5)$ & $45(62.5)$ \\
\hline BMI $\mathrm{kg} \cdot \mathrm{m}^{-2}$ & $30.4 \pm 5.5$ & $31.1 \pm 6.2$ & $29.6 \pm 4.8$ \\
\hline $\mathrm{BMI} \geqslant 30 \mathrm{mg} \cdot \mathrm{m}^{-2}$ & 74 (51.8) & $39(53.4)$ & $35(48.6)$ \\
\hline Neck circumference $\mathrm{cm}$ & $40.9 \pm 3.6$ & $41.4 \pm 3.5$ & $40.3 \pm 3.9$ \\
\hline ESS score & $9 \pm 4$ & $9 \pm 4$ & $9 \pm 4$ \\
\hline ESS score $\geqslant 10$ & $54(37.8)$ & $28(38.4)$ & $26(36.1)$ \\
\hline Sleep study: respiratory polygraphy & $118(82.5)$ & $57(78.1)$ & $61(84.7)$ \\
\hline Arterial hypertension & $100(69.9)$ & 50 (68.5) & 50 (69.4) \\
\hline Diabetes & $41(28.7)$ & $19(26.0)$ & $22(30.6)$ \\
\hline Dyslipidaemia & $83(58.0)$ & $42(57.5)$ & $41(56.9)$ \\
\hline Past CVEs & $28(19.6)$ & 16 (21.9) & $12(16.7)$ \\
\hline Atrial fibrillation & 19 (13.3) & 9 (12.3) & 10 (13.9) \\
\hline COPD & $16(11.2)$ & $12(16.4)$ & $4(5.6)$ \\
\hline Insomnia & 30 (20.9) & 16 (21.9) & $14(19.4)$ \\
\hline Depression & $24(16.8)$ & 10 (13.7) & $14(19.4)$ \\
\hline AHI events $h^{-1}$ & $21.7 \pm 4.8$ & $22.2 \pm 4.3$ & $21.3 \pm 5.2$ \\
\hline ODI3\% events: $\mathrm{h}^{-1}$ & $20.4 \pm 9.9$ & $21.3 \pm 7.8$ & $19.6 \pm 11.7$ \\
\hline$T_{\text {sat }}<90 \% \%$ & $14.4 \pm 25.5$ & $14.2 \pm 28.1$ & $14.6 \pm 22.7$ \\
\hline Central AHI events $\cdot h^{-1}$ & $1.43 \pm 2.5$ & $1.32 \pm 2.4$ & $1.53 \pm 2.6$ \\
\hline Minimum oxygen saturation \% & $78.5 \pm 10.4$ & $77.9 \pm 8.7$ & $79.1 \pm 11.9$ \\
\hline
\end{tabular}

Data are presented as $n$, mean \pm SD or $n(\%)$. CPAP: continuous positive airway pressure; BMI: body mass index; ESS: Epworth Sleepiness Scale; CVE: cardiovascular event lischaemic heart disease and cerebrovascular disease); COPD: chronic obstructive pulmonary disease; AHI: apnoea-hypopnoea index; ODI3\%: oxygen desaturation index at $3 \%$; $T_{\text {sat }}<90 \%$ : night time spent with oxygen saturation $<90 \%$. 
None of the patients presented central sleep apnoea. Mean \pm SD AHI was $21.7 \pm 4.8$ events $\cdot h^{-1}$ (93\% of events were obstructive), which was very similar to that for the oxygen desaturation index (ODI) at $3 \%$ $\left(20.4 \pm 9.9\right.$ events $\left.\cdot h^{-1}\right)$.

Mean \pm SD use of CPAP treatment was $5.2 \pm 2.5 \mathrm{~h} \cdot$ day $^{-1}$, with 20 patients presenting $<4 \mathrm{~h} \cdot$ night $^{-1}(72.6 \%$ with good adherence). Mean \pm SD CPAP pressure used was $7.9 \pm 1.8 \mathrm{cmH}_{2} \mathrm{O}$. Mean \pm sD residual AHI following the application of CPAP during the titration study was $4 \pm 3.2$ events $\cdot \mathrm{h}^{-1}$. No change in BMI was observed between randomised groups over the course of the study (mean $\pm \mathrm{sD} 30.4 \pm 5.5$ versus $30.6 \pm 5.7 \mathrm{~kg} \cdot \mathrm{m}^{-2}$; $\mathrm{p}=0.87$ ). Respiratory polygraphy was performed in $82.5 \%$ of the studies. No baseline differences were observed between those patients under respiratory polygraphy and those under PSG.

\section{Effect on the primary outcome (ESS score) and other sleep-related symptoms}

Mean \pm SD ESS scores were $9.4 \pm 4.1$ and $8.9 \pm 3.7$ at baseline and $5.9 \pm 3.5$ and $8.3 \pm 3.8$ at end of follow-up for the CPAP group (effect size 1) and control group (effect size 0.2), respectively. After adjusting for baseline ESS score, a significant decrease of 2.6 (95\% CI -3.6--1.6) points was observed in the CPAP group compared with the control group. Those patients under CPAP treatment had OR 13.1 (95\% CI 4.7-36.5; $\mathrm{p}<0.0001$ ) of decreasing the ESS score to $<10$ compared with those not treated with CPAP (table 2). There was a positive correlation between the change in ESS scores between groups and the number of hours of CPAP use $(r=0.31 ; p=0.007)$. Although there was an increased dispersion of the ESS score changes in those patients with increased use of CPAP, there were no differences in either age or AHI values between the groups using CPAP $<6 \mathrm{~h} \cdot$ day $^{-1}$ and those using CPAP $>6 \mathrm{~h} \cdot$ day $^{-1}$ (figure 2).

There was, however, a significant improvement in snoring (OR 20.8, 95\% CI 8.6-50.1), witnessed apnoeas (OR 8.7, 95\% CI 3.7-19.9) and choking (OR 3.3, 95\% CI 1.1-12.2), but not in nocturia (OR 0.6, 95\% CI $0.3-1.2$ ) and nightmares (OR 1.3, 95\% CI 0.4-3.8), in the CPAP group (table 2).

\section{Effect on sleep-related quality of life}

Table 3 shows that only the QSQ domains relating to nocturnal symptoms and emotions improved significantly in the CPAP group compared with the control group, adjusting for their corresponding baseline values, especially the nocturnal symptoms with an effect size of 0.86 . There was a positive correlation between the changes between groups in the nocturnal symptoms domain $(r=0.29 ; p=0.01)$, emotions domain $(r=0.33 ; \mathrm{p}=0.005)$ and social interactions domain $(\mathrm{r}=0.35 ; \mathrm{p}=0.003)$ and the number of hours of CPAP use (figure 3).

Effect on anxiety and depression scores and neurocognitive function

$26(17.7 \%)$ patients scored $\geqslant 11$ points in HADS-Depression and $30(20.4 \%)$ patients scored $\geqslant 11$ points in HADS-Anxiety. Table 4 shows that CPAP treatment did not achieve a significant improvement in HADS-Depression and HAS-Anxiety scores or any neurocognitive test.

The TMT-A median (IQR) values were 94 (56-125) and 68.5 (50-132) at baseline and 70 (49-121) and 60 (47-100) at end of follow-up for the CPAP and control groups, respectively. The TMT-B values were 200 (162-300) and $198(127-300)$ at baseline and $186(121-299)$ and 180 (113-300) at end of follow-up for the CPAP and control groups, respectively. No statistical differences were found in the intergroup analysis after adjusting for their corresponding baseline measures $(-5.6$ (95\% CI $-14.3-3)$; $\mathrm{p}=0.2$ and $-9.4(95 \%$ CI -24-5.3); $\mathrm{p}=0.21$ ) for TMT-A and TMT-B, respectively.

TABLE 2 Changes in sleep-related symptoms between randomised groups: intention-to-treat principle, adjusted for baseline measurements

\begin{tabular}{|c|c|c|c|c|c|c|}
\hline & \multicolumn{2}{|c|}{ CPAP treatment ${ }^{\#}$} & \multicolumn{2}{|c|}{ No CPAP treatment" } & \multirow[t]{2}{*}{ OR $\left(95 \% \mathrm{CI}^{+}\right.$} & \multirow[t]{2}{*}{$\mathrm{p}$-value } \\
\hline & Baseline & Follow-up & Baseline & Follow-up & & \\
\hline Snoring & 70 (95.8) & $15(20.5)$ & 66 (91.7) & $58(80.6)$ & $20.8(8.6-50.1)$ & $<0.0001$ \\
\hline Witnessed apnoeas & $53(72.6)$ & $11(15.1)$ & 56 (77.8) & $42(58.3)$ & $8.7(3.7-19.9)$ & $<0.0001$ \\
\hline Choking & $18(24.7)$ & $4(5.5)$ & $12(16.7)$ & 9 (12.5) & $3.3(1.1-12.2)$ & 0.041 \\
\hline Nocturia & $41(56.2)$ & $31(42.5)$ & 39 (54.2) & 23 (31.9) & $0.6(0.3-1.2)$ & 0.109 \\
\hline Nightmares & 15 (20.5) & $9(12.3)$ & $14(19.4)$ & $10(13.9)$ & $1.3(0.4-3.8)$ & 0.68 \\
\hline ESS score $\geqslant 10$ & $28(38.4)$ & $8(10.9)$ & $26(36.1)$ & 23 (31.9) & $13.1(4.7-36.5)$ & $<0.0001$ \\
\hline
\end{tabular}

Data are presented as $\mathrm{n}(\%)$, unless otherwise stated. CPAP: continuous positive airway pressure; ESS: Epworth Sleepiness Scale. ${ }^{\#}$ : $n=73 ;{ }^{\uparrow}: n=72 ;^{+}$: adjusted for baseline data. 
FIGURE 2 Correlation between intergroup changes in the Epworth Sleepiness Scale (ESS) score and the number of hours of continuous positive airway pressure (CPAP) use.

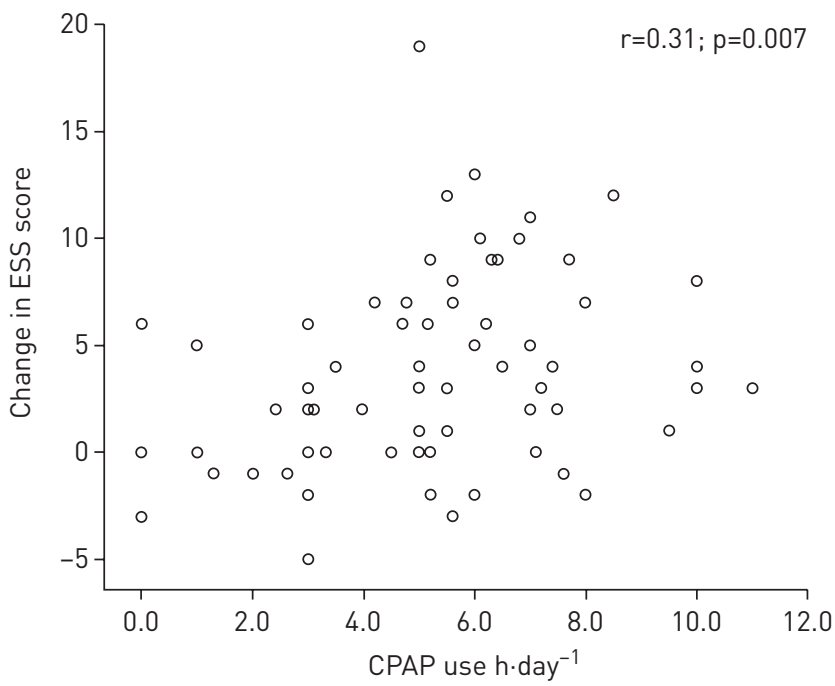

Effect on office-based blood pressure levels

Finally, no differences were observed in changes in either office-based systolic blood pressure $(-0.9$ versus $-0.2 \mathrm{mmHg} ; \mathrm{p}=0.9)$ or diastolic blood pressure $(-1.3$ versus $-1.2 \mathrm{mmHg} ; \mathrm{p}=0.8)$ measurements when comparing the CPAP and control groups.

\section{Per-protocol analysis}

A per-protocol analysis was also perfomed by comparing those patients with good adherence to CPAP treatment with the control group without CPAP. When those patients who tolerated CPAP for at least 4 h.day ${ }^{-1}(n=53)$ were compared with the control group without CPAP $(n=72)$, a statistically significant difference was observed. The results are given in the supplementary material.

\section{Discussion}

This study develops and expands previous trials in older people by evaluating the efficacy of CPAP treatment in elderly patients with moderate to severe OSA. According to our results, CPAP improved the clinical picture related to OSA, including hypersomnia, as well as some aspects of the quality of life, without any changes in the neurocognitive tests or blood pressure measurements.

Only two clinical trials on the effect of CPAP on exclusive series of elderly OSA patients have been published to date. MCMillan et al. [7] observed 12 months of CPAP treatment in patients aged $\geqslant 65$ years with OSA of any degree of severity: although they found an improvement in the degree of hypersomnia ( 2.1 points in the ESS score at 3 months and 2 points at 12 months), this was not the case in variables linked to the cost-effectiveness of treatment, quality-of-life, neurocognition and blood pressure measurements. For their part, MarTínez-García et al. [6] observed a significant improvement in clinical symptoms (the ESS score improved by 3.4 points), quality of life and some neurocognitive tests associated with working memory in patients aged $\geqslant 70$ years with a diagnosis of severe OSA (AHI $\geqslant 30$ events $\cdot \mathrm{h}^{-1}$ ).

TABLE 3 Changes in sleep-related quality of life (Quebec Sleep Questionnaire domains) between randomised groups: intention-to-treat principle, adjusted for baseline measurements

\begin{tabular}{|c|c|c|c|c|c|c|c|c|c|c|}
\hline & \multicolumn{4}{|c|}{ CPAP treatment ${ }^{\#}$} & \multicolumn{4}{|c|}{ No CPAP treatment ${ }^{\pi}$} & \multirow{2}{*}{$\begin{array}{l}\text { Intergroup } \\
\text { difference } \\
\left(95 \% \mathrm{CI}^{+}\right.\end{array}$} & \multirow[t]{2}{*}{ p-value } \\
\hline & $\begin{array}{c}\text { Baseline } \\
\text { score }\end{array}$ & $\begin{array}{l}\text { Follow-up } \\
\text { score }\end{array}$ & $\begin{array}{l}\text { Effect } \\
\text { size }\end{array}$ & $\begin{array}{l}\text { Intragroup } \\
\text { difference }\end{array}$ & $\begin{array}{l}\text { Baseline } \\
\text { score }\end{array}$ & $\begin{array}{l}\text { Follow-up } \\
\text { score }\end{array}$ & $\begin{array}{l}\text { Effect } \\
\text { size }\end{array}$ & $\begin{array}{l}\text { Intragroup } \\
\text { difference }\end{array}$ & & \\
\hline Hypersomnolence & $5.5 \pm 1.3$ & $5.9 \pm 1.2$ & 0.31 & $-0.4 \pm 1.3$ & $5.7 \pm 1.1$ & $5.8 \pm 1.3$ & 0.10 & $-0.1 \pm 1.0$ & $-0.3(-0.6-0.1)$ & 0.12 \\
\hline Diurnal symptoms & $5.0 \pm 1.5$ & $5.5 \pm 1.3$ & 0.33 & $-0.5 \pm 1.2$ & $5.2 \pm 1.4$ & $5.3 \pm 1.3$ & 0.10 & $-0.1 \pm 0.9$ & $-0.3(-0.6--0.1)$ & 0.12 \\
\hline Social interactions & $5.6 \pm 1.4$ & $6.1 \pm 0.9$ & 0.29 & $-0.4 \pm 1.5$ & $5.8 \pm 1.2$ & $6.0 \pm 1.1$ & 0.16 & $-0.2 \pm 0.9$ & $-0.1(-0.4-0.2)$ & 0.38 \\
\hline
\end{tabular}

Data are presented as mean \pm SD, unless otherwise stated. CPAP: continuous positive airway pressure. ${ }^{\#}: \mathrm{n}=73 ;{ }^{\text {9}}: \mathrm{n}=72 ;{ }^{+}$: adjusted for baseline data. 

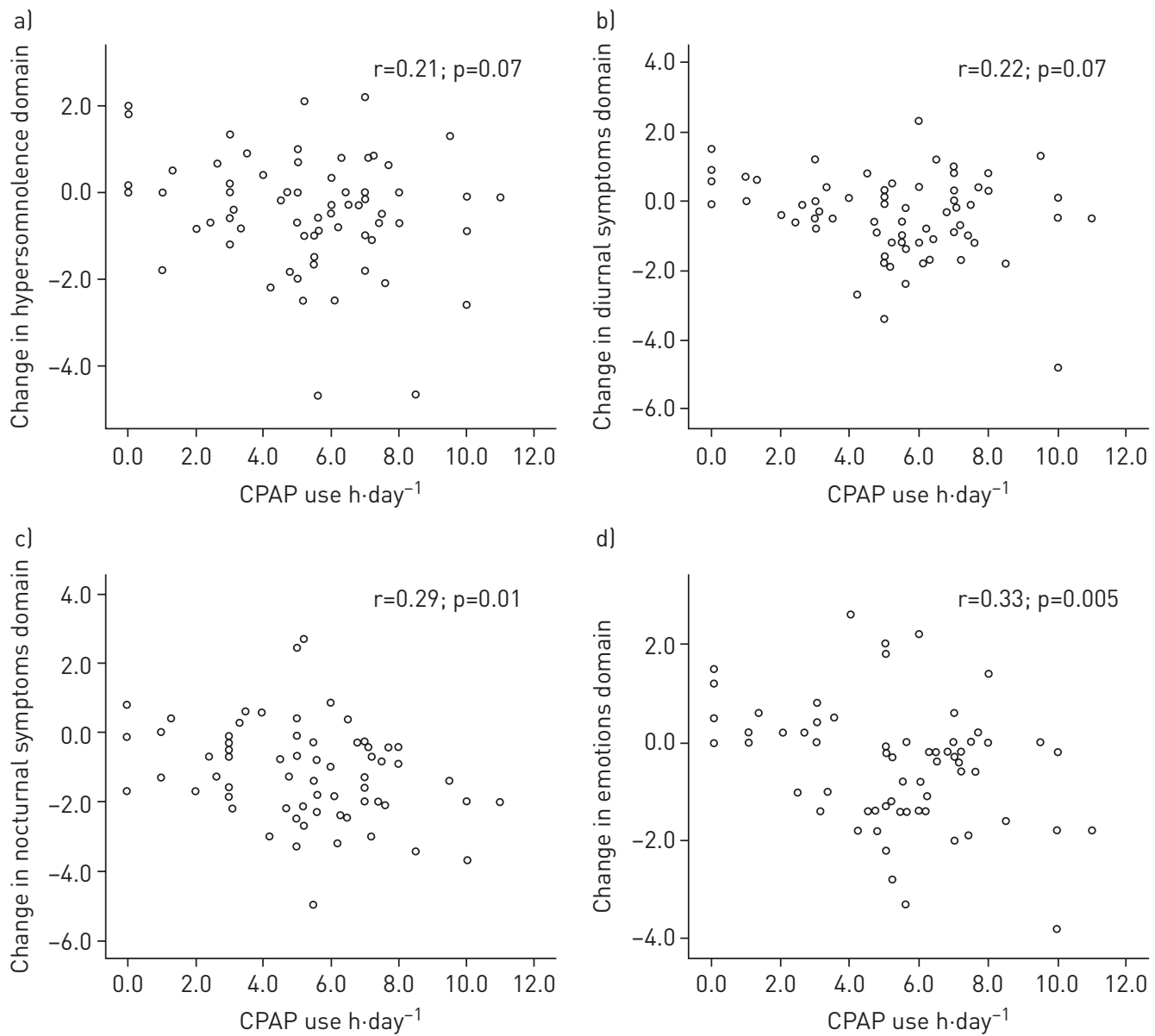

d)
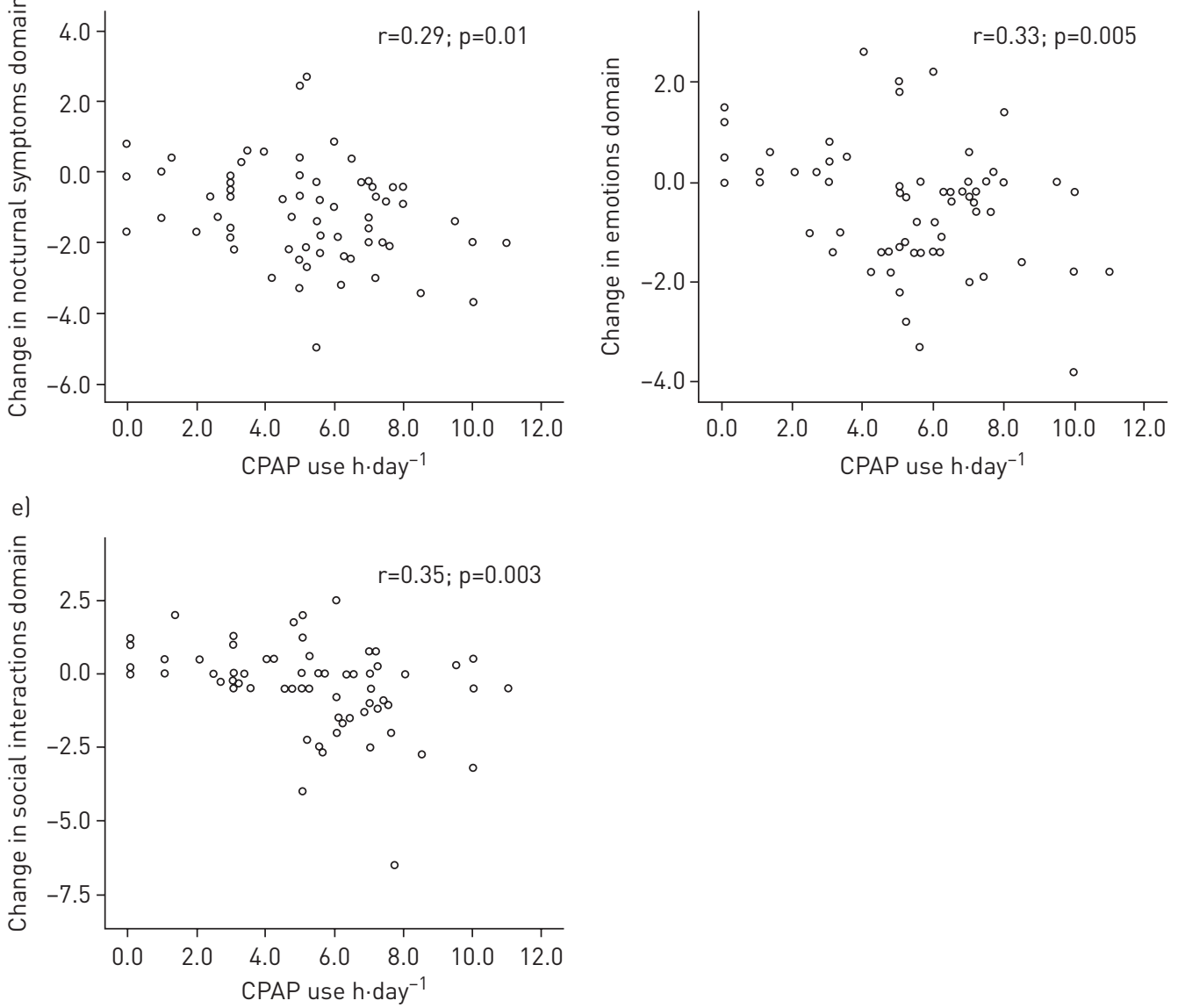

FIGURE 3 Correlation between intergroup changes in the Quebec Sleep Questionnaire domains and the number of hours of continuous positive airway pressure (CPAP) use: a) hypersomnolence, b) diurnal symptoms, c) nocturnal symptoms, d) emotions and e) social interactions.

As the number of sleep-related respiratory events increases with age for physiological reasons $[4,5,18-20]$, and as we do not know the AHI value that distinguishes the physiological from the pathological, it seemed reasonable to perform a randomised controlled trial similar to the one performed by our group on severe OSA, using the same variables and a diagnostic method that would make it possible to calculate both the ODI and AHI (respiratory polygraphy or PSG) in elderly patients with moderate OSA (defined as AHI 15-29.9 events. ${ }^{-1}$ ) [8]. This also allowed us to make a direct comparison of the effects of CPAP on the results of the two clinical trials (severe OSA versus moderate OSA).

Although in the present study the effect on the main variable (ESS score) improved on both a statistically and clinically significant basis, in keeping with the minimum important difference of 2 points recently 
TABLE 4 Changes in neurocognitive tests between randomised groups: intention-to-treat principle, adjusted for baseline measurements

CPAP treatment ${ }^{\#}$

No CPAP treatment ${ }^{\text {T }}$

Intergroup $\quad \mathrm{p}$-value
difference $\left(95 \% \mathrm{CI}^{+}\right.$

\begin{tabular}{|c|c|c|c|c|c|c|c|c|c|c|}
\hline & & & & & & & \\
\hline & $\begin{array}{l}\text { Baseline } \\
\text { score }\end{array}$ & $\begin{array}{l}\text { Follow-up } \\
\text { score }\end{array}$ & $\begin{array}{l}\text { Effect } \\
\text { size }\end{array}$ & $\begin{array}{l}\text { Intragroup } \\
\text { difference }\end{array}$ & $\begin{array}{l}\text { Baseline } \\
\text { score }\end{array}$ & $\begin{array}{l}\text { Follow-up } \\
\text { score }\end{array}$ & $\begin{array}{l}\text { Effect } \\
\text { size }\end{array}$ & $\begin{array}{l}\text { Intragroup } \\
\text { difference }\end{array}$ & & \\
\hline HADS-Anxiety & $6.6 \pm 4.4$ & $5.7 \pm 4.5$ & 0.20 & $0.9 \pm 3.5$ & $6.1 \pm 4.9$ & $6.1 \pm 4.9$ & 0.00 & $0.0 \pm 2.4$ & $0.8(0.2-1.7)$ & 0.12 \\
\hline Digit span test & $8.3 \pm 4.0$ & $8.6 \pm 2.1$ & 0.10 & $-0.3 \pm 3.5$ & $8.4 \pm 2.7$ & $8.3 \pm 2.6$ & 0.03 & $0.1 \pm 1.6$ & $-0.2(-0.6-0.9)$ & 0.63 \\
\hline Digit symbol test & $21.2 \pm 11.1$ & $23.2 \pm 11.5$ & 0.20 & $-2.0 \pm 6.3$ & $20.4 \pm 10.0$ & $21.7 \pm 11.2$ & 0.10 & $-1.3 \pm 7.2$ & $-0.9(-2.5-4.3)$ & 0.59 \\
\hline
\end{tabular}

Data are presented as mean \pm SD, unless otherwise stated. CPAP: continuous positive airway pressure; HADS: Hospital Anxiety and Depression Scale. ${ }^{\#}: \mathrm{n}=73 ;{ }^{\uparrow}: \mathrm{n}=72 ;^{+}$: adjusted for baseline data.

calculated by Своок et al. [20], this improvement (2.6 points) was not as substantial as the improvement attained with severe OSA (3.4 points). This difference is probably due to the greater degree of baseline hypersomnia present with severe OSA as well as the probable greater aetiological responsibility of severe OSA for hypersomnia compared with moderate OSA. With regard other sleep-related symptoms, there was a similar improvement in severe OSA, although to a lesser extent, but there was no statistical difference in the incidence of nightmares or the degree of nocturia (although nocturia did significantly improve in those patients with good CPAP compliance). Maybe these two symptoms are less closely related to OSA itself in moderate cases, but instead have a greater connection with other comorbidities common to elderly people.

One basic difference between our previous study [6] and the current study is that all quality of life domains improved with CPAP in severe OSA patients, whereas only two did so in moderate OSA patients (nocturnal symptoms and emotions). This could concur with the improvements seen in the energy and vitality domains of the 36-item Short-Form Health Survey questionnaire in the McMilLan et al. [7] study. However, the improvement was much more substantial in all the domains in the per-protocol analysis, as this was only applied to patients with good CPAP compliance. It is possible that elderly patients with moderate OSA need more hours of CPAP to improve their quality of life than their counterparts with severe OSA, as the latter's quality of life is more markedly impacted by the disease [21-23] and is therefore more susceptible to improvement with CPAP. It is worth noting that although all the domains of the QSQ presented a significant statistical improvement with CPAP compared with the control group, they did not reach the minimum clinically significant difference established by the authors of the QSQ [11]. However, it is important to bear in mind that the clinically significant differences of the QSQ were validated in a population with a mean age of 55 years, i.e. 20 years lower than that of the patients included in the present study. Therefore, these minimum differences may not be applicable to the present study.

Another fundamental difference between the two studies was the lack of improvement (even in the per-protocol analysis) in the questionnaires on anxiety and depression in moderate OSA, in contrast to the results in severe OSA, where there was a clear improvement even in the ITT analysis. The explanation for this phenomenon could be similar to that for the improvement in other symptoms like nocturia and nightmares, since it is possible that the presence of anxiety and depression (both very common in the elderly [24]) is more closely associated with severe OSA than with moderate OSA, and so an improvement with CPAP would be more likely in the former case.

Similarly, it was not possible to corroborate the neurocognitive improvement observed in severe OSA in moderate OSA, even in the per-protocol analysis. This could be because only a low percentage of patients with moderate OSA and neurocognitive deficits are seen in the consulting room and are therefore susceptible to improvement. Accordingly, when patients with this type of deficit (such as those associated with Alzheimer's disease) have been included, some studies have demonstrated a significant improvement with CPAP $[25,26]$.

Finally, the initial level (at 3 months) of compliance with CPAP was good $(>70 \%)$ in moderate OSA patients, with at least $4 \mathrm{~h} \cdot \mathrm{day}^{-1}$; this was very similar to the measurements for severe OSA, which shows that CPAP use at this age is not very different to that observed in middle-aged patients, in contrast to the results of some other authors $[22,23]$.

Among the strengths of the present study, apart from the methodology of the multicentre clinical trial used, is the application of a methodology similar to that of the previous study conducted with elderly 
patients with severe OSA by our own group [6], which enabled us to directly compare the results of the two groups. Furthermore, there were very few lost patients or missing data.

The limitations of the study include the fact that $75 \%$ of the sleep tests were conducted with home polygraphy and 25\% with full PSG. This situation was tolerated in order to be able to include hospitals with limited access to PSG. In any case, no patients were selected beforehand for one test or the other; this choice depended entirely on the equipment available in each hospital. Moreover, a comparison of the baseline variables of those patients who received PSG and those who received respiratory polygraphy did not produce any statistically different results. Another potential limitation of the study is the absence of a placebo group receiving sham-CPAP, although in our opinion sham-CPAP is not a true placebo, as several authors have indicated $[27,28]$. Finally, only 20 out of the 144 patients were aged $>80$ years, so the results in very elderly people cannot be inferred from this study.

In conclusion, in patients aged $\geqslant 70$ years referred to sleep units on suspicion of OSA, CPAP treatment significantly reduces the degree of hypersomnia and other sleep-related symptoms, along with improvements in some health-related quality of life domains, but it has no effect on neurocognitive variables, blood pressure measurements or the degree of anxiety and depression. The improvement in the quality of life largely depends on good compliance with CPAP and so elderly patients selected for CPAP treatment must be closely monitored in this respect.

Author contributions: S. Ponce and M.Á. Martínez-García participated in study conception and design, supervised the study, and wrote the first manuscript draft, with input from all of the authors. A. Muriel participated in data interpretation. E. Pastor, P. Catalán, A. Martinez, L. Hernández, B. Orosa, G. Oscullo and E. Chiner participated in data collection. All of the other authors contributed to data acquisition and interpretation, critically revised the manuscript, and made the decision to submit the manuscript for publication and approved the final version for publication. All of the authors take responsibility for the accuracy and completeness of the data and analyses reported, and for the fidelity of the studies to the protocols.

Conflict of interest: None declared.

Support statement: This work was supported by the Fundación Valenciana de Neumología.

\section{References}

1 Christensen K, Doblhammer G, Rau R, et al. Ageing populations: the challenges ahead. Lancet 2009; 374: $1196-1208$.

2 Levy P, Kohler M, McNocholas WT, et al. Obstructive sleep apnoea syndrome. Nat Rev 2015; 1: 1-20.

3 Martinez-García MA, Amibilia J, Chiner E, et al. Sleep apnea in patients of elderly: care activity in Spain (20022008). Arch Bronconeumol 2010; 46: 502-507.

4 Netzer NC, Ancoli-Israel S, Bliwise DL, et al. Principles of practice parameters for the treatment of sleep disordered breathing in the elderly and frail elderly: the consensus of the International Geriatric Sleep Medicine Task Force. Eur Respir J 2016; 48: 992-1018.

5 Bixler EO, Vgontzas AN, Ten Have T, et al. Effects of age on sleep apnea in men. Prevalence and severity. Am J Respir Crit Care Med 1998; 157: 44-148.

6 Martínez-García MA, Chiner E, Hernández L, et al. Obstructive sleep apnoea in the elderly: role of continuous positive airway pressure treatment. A randomised, controlled trial. Eur Respir J 2015; 46: 142-151.

7 McMillan A, Bratton DJ, Faria R, et al. Continuous positive pressure in the respiratory tract in elderly people with obstructive sleep apnea syndrome: a randomized, multicenter, 12-month trial. Lancet Respir Med 2014; 2: 804-814.

8 American Academy of Sleep Medicine Task Force. Sleep-related breathing disorders in adults: recommendations for syndrome definition and measurement techniques in clinical research. Sleep 1999; 22: 667-689.

9 Lloberes P, Durán-Cantolla J, Martínez-García MA, et al. Diagnosis and treatment of sleep apnea-hypopnea syndrome. Arch Bronconeumol 2011; 47: 143-156.

10 Chiner E, Arriero JM, Signes-Costa J, et al. Validation of the Spanish version of the Epworth Sleepiness Scale in patients with sleep apnea syndrome. Arch Bronconeumol 1999; 35: 422-427.

11 Lacasse Y, Bureau MP, Sériès F. A new standardised and self-administrated quality of life questionnaire specific to obstructive sleep apnoea. Thorax 2004; 59: 494-499.

12 Catalán P, Martínez A, Herrerón A, et al. Internal consistency and validity of the Spanish version of the "Quebec Sleep Questionnaire” quality-of-life questionnaire for obstructive sleep apnea. Arch Bronconeumol 2012; 48: 107-113.

13 Lezack MD. Neuropsychological Assessment. 3rd Edn. New York, Oxford University Press, 1995.

14 Wechsler DA. Wechsler Adults Intelligence Scale (WAIS). Madrid, Tea Ediciones, 1976.

15 Zigmond AS, Snaith RP. The hospital anxiety and depression scale. Acta Psychiatr Scand 1983; 67: 361-370.

16 The Task Force for the Management of Arterial Hypertension of the European Society of Hypertension (ESH) and of the European Society of Cardiology (ESC). 2007 Guidelines for the Management of Arterial Hypertension. J Hypertens 2007; 25: 1105-1187.

17 Cohen J. Statistical Power Analysis for the Behavioral Sciences. NewYork, Academic Press, 1969.

18 Duran J, Esnaola S, Rubio R, et al. Obstructive apnea-hypopnea and related clinical features in a population-based sample of subjects aged 30 to 70 yr. Am J Respir Crit Care Med 2001; 163: 685-689. 
19 Martinez-Garcia MA, Duran-Cantolla J, Monserrat JM. Sleep apnea-hypopnea syndrome in elderly. Arch Bronconeumol 2012; 46: 479-488.

20 Crook S, Sievi NA, Bloch KE, et al. Minimum important difference of the Epworth Sleepiness Scale in obstructive sleep apnoea: estimation from three randomized controlled trials. Thorax 2019; 74: 390-396.

21 Martinez-Garcia MA, Soler JJ, Román P, et al. Obstructive sleep apnea has little impact on quality of life in the elderly. Sleep Med 2009; 10: 104-111.

22 Weaver TE, Chasens ER. Continuous positive airway pressure treatment for sleep apnea in older adults. Sleep Med Rev 2007; 11: 99-111.

23 Weaver TE, Grunstein RR. Adherence to continuous positive airway pressure therapy: the challenge to effective treatment. Proc Am Thorac Soc 2008; 5: 173-178.

24 Saunamaki T, Jehkonen M. Depression and anxiety in obstructive sleep apnea syndrome: a review. Acta Neurol Scand 2007; 116: 277-288.

25 Yaffe K, Laffan AM, Harrison SL, et al. Sleep-disordered breathing, hypoxia, and risk of mild cognitive impairment and dementia in older woman. JAMA 2011; 306: 613-619.

26 Ancoli-Israel S, Palmer BW, Cooke JR, et al. Cognitive effects of treating obstructive sleep apnea in Alzheimer's disease: a randomized controlled study. J Am Geriatr Soc 2008; 56: 2076-2081.

27 Hein H. Is sham CPAP a true placebo? Am J Respir Crit Care Med 2002; 165: 305.

28 Schwartz SW, Cimino CR, Anderson WM. CPAP or placebo-effect? Sleep 2012; 35: 1585-1586. 